\title{
Humanistic and economic impact of subcutaneous versus intravenous administration of oncology biologics
}

\author{
Kenneth C Anderson ${ }^{1}$, Ola Landgren², Rebecca C Arend ${ }^{3}$, Jeffrey Chou ${ }^{4}$ \& Ira A Jacobs ${ }^{*}, 5$ \\ ${ }^{1}$ Division of Hematologic Neoplasia, Harvard Medical School, Dana-Farber Cancer Institute, Boston, MA 02215, USA \\ ${ }^{2}$ Myeloma Service, Memorial Sloan Kettering Cancer Center, New York, NY 10065, USA \\ ${ }^{3}$ University of Alabama at Birmingham, Comprehensive Cancer Center, Experimental Therapeutics Program, Birmingham, AL \\ 35294, USA \\ ${ }^{4}$ Pfizer, San Francisco, CA 94080, USA \\ ${ }^{5}$ Pfizer, New York, NY 10017, USA \\ *Author for correspondence: Tel.: +1 212733 0876; Fax: +1 845474 5301; ira.jacobs@pfizer.com
}

More oncology biologics are becoming available for subcutaneous (sc.) administration and are expected to provide useful therapeutic options. We evaluated evidence published in the past 5 years to assess the humanistic and economic impact of sc. versus intravenous administration of approved cancer therapies and identify outcomes favoring either administration route. These publications focused predominantly on healthcare resource utilization and economic outcomes, demonstrating resource and cost savings with sc. administration. Patients reported a better health-related quality of life and preference for sc. formulations. Time-and-motion study analyses confirmed the convenience of sc. administration. These findings suggest that future availability of sc. oncology biologics, especially anti-PD-1/PD-L1 antibodies due to their increased utility in various malignancies, may be beneficial for patients, healthcare providers and payers.

First draft submitted: 26 June 2019; Accepted for publication: 25 July 2019; Published online:

9 August 2019

Keywords: biologic $\bullet$ cancer $\bullet$ cost $\bullet \mathrm{HCRU} \bullet \mathrm{HRQOL} \bullet$ intravenous $\bullet$ preference $\bullet$ subcutaneous $\bullet$ tolerability

Several systemic biologic therapies are currently available in multiple formulations for intravenous (iv.), subcutaneous (sc.) or other routes of administration [1-3]. While efficacy and safety for each drug are expected to be similar across their approved formulations and administration routes, each route may have certain advantages in terms of convenience, patient preference, compliance, administration time, direct/indirect costs and other factors. Oncology biopharmaceuticals available in sc. formulations that can replace iv. therapies are of particular interest for healthcare providers (HCPs), patients and payers as they may contribute to improve health-related quality of life (HRQoL), optimize oncology healthcare resource utilization (HCRU) and minimize costs of cancer treatments [1,2].

Oncology agents with both iv. and sc. formulations approved for the treatment of patients with solid tumors or hematologic malignancies, currently include the anti-CD20 antibody rituximab, the anti-HER2 antibody trastuzumab and the proteasome inhibitor bortezomib [4-6]. Other sc. formulations are under investigation for biologic agents, including PD-1/PD-L1 inhibitors, pertuzumab, bevacizumab and daratumumab [7-12]. Once approved, the availability of an increasing number of oncology agents in sc. as well as iv. formulations would expand options for patients and HCPs, and could have positive implications for treatment.

Although intuitively sc. administration is expected to provide advantages compared with iv. administration, to date there are limited published data analyses on tolerability, patient preferences, HCRU and costs associated with sc. versus iv. administration of oncology drugs across multiple approved agents. Thus, the objective of this study was to evaluate and compare evidence from the published literature on the humanistic and economic impact of sc. versus iv. administration of biologics approved for the treatment of patients with solid and hematologic malignancies. 


\section{Methods}

A systematic rapid evidence assessment (REA) methodology was used to identify, review and report the most relevant literature published in the past 5 years, adhering to the Preferred Reporting Items for Systematic Reviews and Meta-Analyses protocol (PRISMA-P) guidelines [13], as applicable. We selected a 5-year interval for this REA so that the included data were not outdated, while ensuring a reasonably sized pool of studies for evaluation.

\section{Publication selection}

The REA methodology entailed a rigorous and objective search/selection process following prespecified inclusion/exclusion criteria, to capture the most relevant oncology publications (search strategy shown in Supplementary Table 1).

\section{Literature databases}

The following databases were searched to identify the most relevant peer-reviewed publications that reported a cancer-related term (i.e., cancer, neoplasm, carcinoma, lymphoma, myeloma) and both iv. and sc. treatment terms and a biologic agent term (i.e., rituximab, trastuzumab): Embase ${ }^{\circledR}$ via Ovid, MEDLINE ${ }^{\circledR}$ (to include in-process citations) via Ovid, Cochrane Central Register of Controlled Trials, Database of Systematic Reviews, Database of Abstracts of Reviews of Effects, Health Technology Assessments, NHS Economic Evaluation Database via Ovid (Figure 1).

Additional searches were performed including: PubMed (most recent year, to capture recent publications not referenced in MEDLINE or Embase), online Congress abstract searches (last 2 years), for American Society of Clinical Oncology, International Society for Pharmacoeconomics and Outcomes Research and Academy of Managed Care Pharmacy. Furthermore, additional publications were identified through searches conducted on reference lists from review articles (Figure 1).

\section{Inclusion criteria}

Studies meeting the following PICOS selection criteria were included: population - adults receiving a biologic agent for a solid or hematologic malignancy; intervention - anticancer biologic, approved and available (in any country) for both sc. and iv. administration (both formulations reported in the same study); comparison/study design - prospective or retrospective, observational studies or clinical trials (Phase $\geq$ II) comparing outcomes for iv. versus sc. formulations of the same biologic agent; outcomes: tolerability (assessed as adverse events [AEs], including reactions related to the administration route), any type of HRQoL outcome directly related to administration route, travel/wait time for patients and caregivers, patient and HCP preferences, time-and-motion outcomes (including preparation/administration efficiency or accuracy, administration time, chair time), HCRU and direct or indirect costs; and other criteria: published in English within the past 5 years $(2$ years for congress abstracts, 1 year for PubMed).

\section{Exclusion criteria}

Studies meeting the following criteria were excluded from the REA: in benign tumors/hematologic disorders or not in oncology; biologic interventions not used directly in cancer treatment (supportive care agents such as filgrastim, pegfilgrastim, sargramostim, epoetin, darbepoetin or biosimilars of these agents); biologic agents used to treat bone metastases (i.e., denosumab and zoledronic acid); only clinical data reported; animal, preclinical or case studies; early/Phase I studies; studies conducted in pediatric populations; reviews, meta-analyses, notes, case reports, comments, editorials, letters or opinions; and publications not in English.

\section{Publication screening \& quality assurance}

At the level 1 screen, a reviewer screened the titles of publications identified in the searches for eligibility according to the inclusion/exclusion criteria. At the level 2 screen, a reviewer screened the full-text publications and abstracts selected at level 1 against the same inclusion/exclusion criteria. In both level 1 and 2 screens, a second reviewer performed a quality check of $10 \%$ of all screened studies. In case of discrepancies, this second reviewer checked all the studies that had been excluded for the same reason. Studies for which there was uncertainty about inclusion were evaluated by a senior researcher. 
(A)

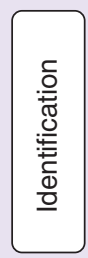

Records identified through database

searches

$(\mathrm{n}=626)$

[Embase, $n=402$; Medline, $n=82$;

Cochrane, $n=119$, PubMed, $n=23$ ]

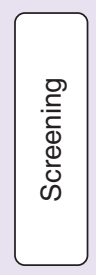

Records after duplicates removed ( $n=450)$ [L1 screening]
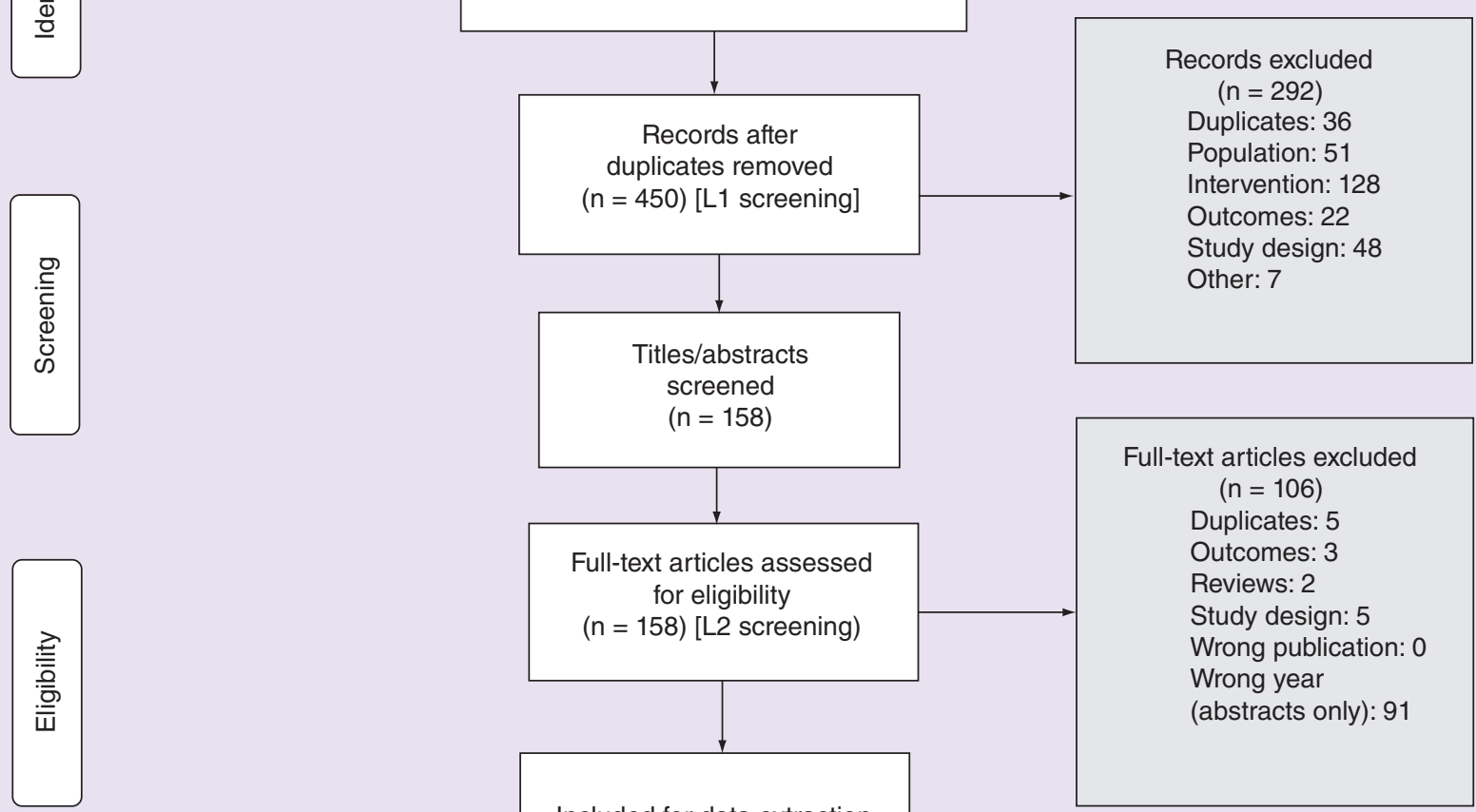

screened

$(n=158)$

Full-text articles excluded $(n=106)$

Duplicates: 5

Outcomes: 3

Reviews: 2

Study design: 5

Wrong publication: 0

Wrong year

(abstracts only): 91

( $n=158)$ [L2 screening)

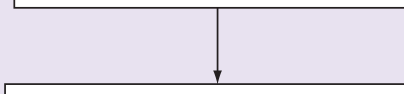

Included for data extraction

$$
(n=52)
$$
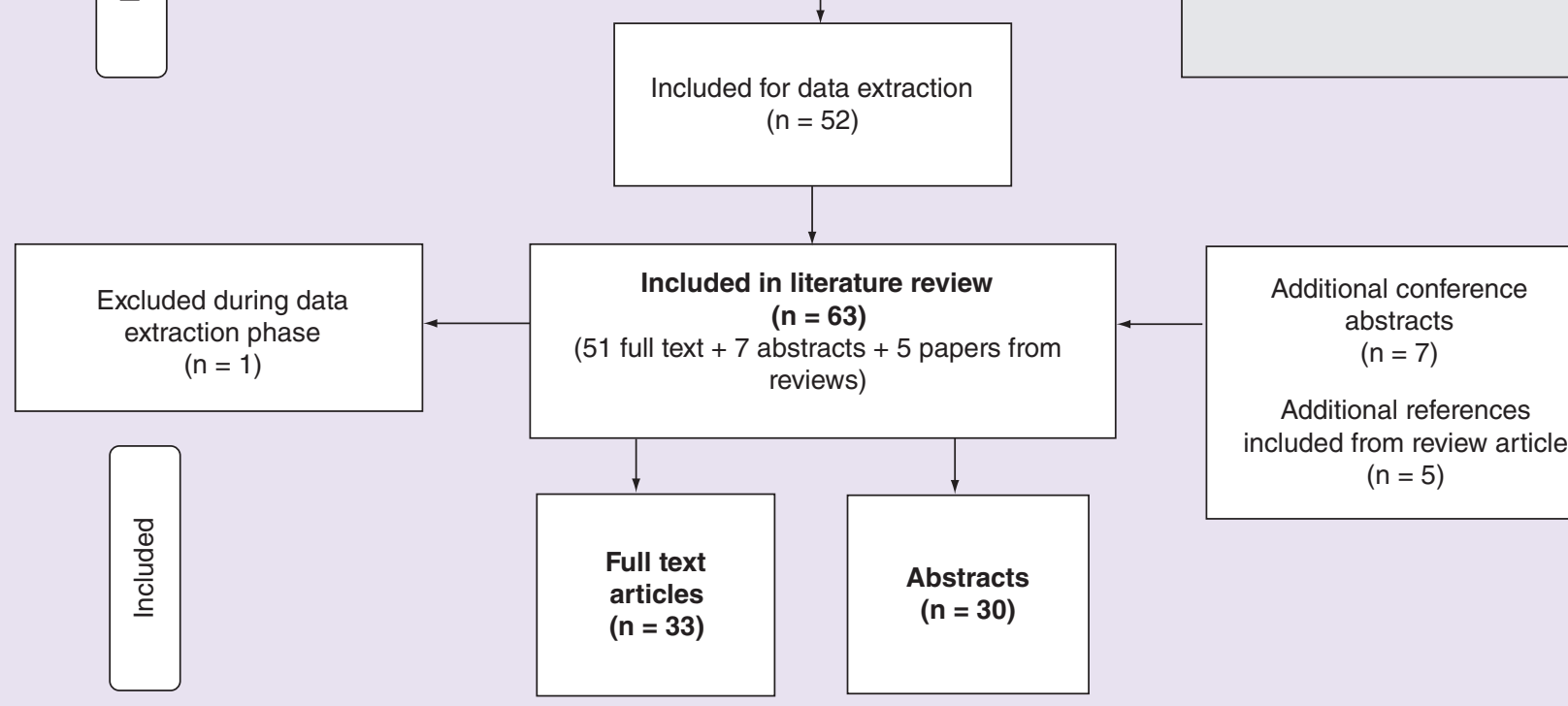

Additional conference abstracts $(\mathrm{n}=7)$

Additional references included from review article $(n=5)$

Figure 1. Preferred reporting items for systematic reviews and meta-analyses protocol flow diagrams. (A) Database publications and (B) conference abstracts.

AMCP: Academy of Managed Care Pharmacy; ASCO: American Society of Clinical Oncology; ISPOR: International Society for Pharmacoeconomics and Outcomes Research; PRISMA: Preferred reporting items for systematic reviews and meta-analyses. 
(B)
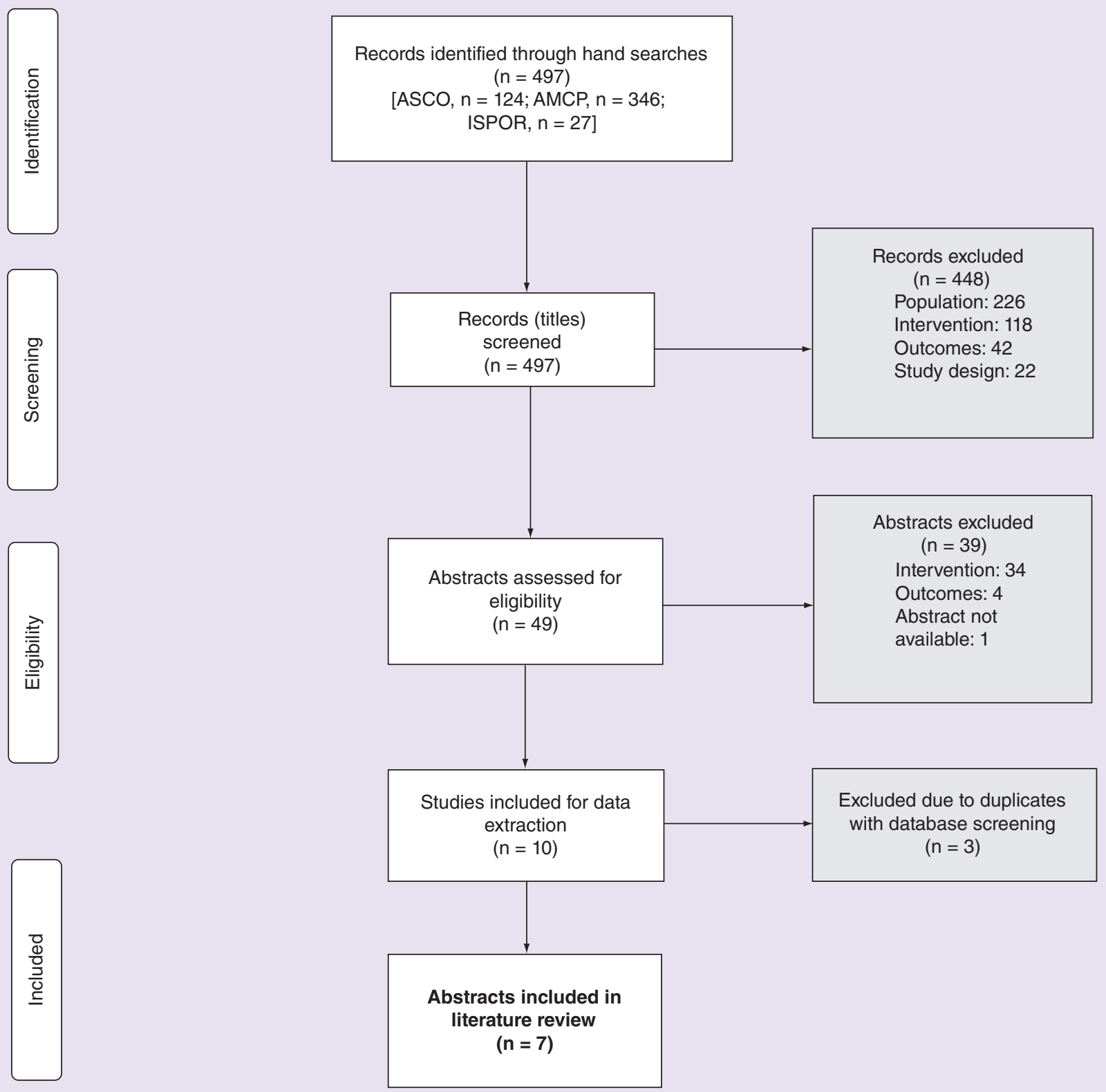

Figure 1. Preferred reporting items for systematic reviews and meta-analyses protocol flow diagrams (cont.). (A) Database publications and (B) conference abstracts.

AMCP: Academy of Managed Care Pharmacy; ASCO: American Society of Clinical Oncology; ISPOR: International Society for Pharmacoeconomics and Outcomes Research; PRISMA: Preferred reporting items for systematic reviews and meta-analyses.

\section{Data extraction \& analysis}

After selecting publications that met inclusion criteria, based on full-text screening, the data elements (Supplementary Table 2) were reported in data extraction tables. All extracted data were validated for accuracy by a separate researcher and analyzed to identify key findings. To determine whether a study outcome favored sc. or iv. administration, p-values or statistical significance were used when reported in a publication. In cases where statistical significance was not available, determinations were based on the results and conclusions of the study. 


\section{Results}

Through database searches and hand screening of reference lists, we identified 450 publications (full articles and congress abstracts), which were screened against the inclusion criteria after removal of study report duplicates (Figure 1). A total of 63 publications (33 manuscripts and 30 congress abstracts) comparing outcomes of sc. versus iv. administration of oncology biologics were retained for this analysis [14-76].

\section{Country of publication, reported therapy \& study type}

Data were reported from 24 countries. Overall, $22 \%$ of the publications (13 manuscripts and 1 abstract) presented data on sc. versus iv. oncology biologics from global, multicountry studies, while $39 \%$ of the publications (13 manuscripts and 12 abstracts) were from Europe. Only one manuscript reported data from a study conducted in the USA. The study country was not specified in three manuscripts and seven congress abstracts.

Three oncology agents were reported within these studies: trastuzumab, rituximab and bortezomib (Table 1 and Supplementary Table 3). The majority of the publications (55\% of manuscripts and $57 \%$ of abstracts) included results for trastuzumab, used for the treatment of patients with HER2-positive breast cancer.

Most publications provided reasonable details on the types of modeling approaches used for analysis. The majority of the manuscripts were prospective clinical trial reports (33\%), primarily from Phase III trials (24\%) (Table 1), while economic models were the most frequent study type among the abstracts (40\%) (Supplementary Table 3).

\section{Reported outcomes}

Many publications reported multiple outcomes, which were grouped for this analysis into tolerability, HRQoL/patient-reported outcomes, HCRU and economic outcomes (Supplementary Table 2). Tolerability was fairly evenly reported in both manuscripts (30\%) and abstracts (21\%), while HRQoL/patient-reported outcomes were the least frequently reported outcomes in manuscripts (16\%) and abstracts (11\%) (Figure 2). HCRU was often analyzed in both manuscripts (34\%) and abstracts (27\%), and it included outcomes identified in time-and-motion studies such as HCP time, chair time and drug preparation/administration time.

Reported outcomes typically included direct medical costs (94\% of publications) involved in patient chair time (i.e., cost of drugs and consumables) and other types of HCRU. Economic outcomes, including primarily direct medical costs or drug administration costs, with few studies reporting indirect costs, were the most frequent outcomes reported in abstracts (46\%).

\section{Analysis of outcomes}

We then analyzed the publications by outcome type to identify which studies favored sc. or iv. administration of approved oncology biologics. Among 61 outcomes compared in 33 manuscripts, 47 (77\%) favored sc., 2 (3\%) favored iv. and $12(20 \%)$ showed no statistically significant difference between sc. and iv. administration (Table 1). In manuscripts, HRQoL, HCRU and economic outcomes consistently favored sc. over iv. administration, with just two of the economic outcomes showing no significant difference between sc. and iv. administration (Figure 2). Among 56 outcomes compared in 30 congress abstracts, 50 (89\%) favored sc., 1 (2\%) favored iv. and 5 (9\%) showed no statistically significant difference between sc. and iv. administration. As with manuscripts, most of the outcomes reported in congress abstracts favored sc. over iv. administration for HRQoL, HCRU and economic outcomes; only one outcome (HCRU) assessed in congress abstracts favored iv. (Supplementary Table 3).

\section{Tolerability outcomes}

Overall, 18 manuscripts and 12 abstracts reported tolerability. Results from 15 (50\%) studies demonstrated no significant difference; 13 (43\%) favored the sc. route; and 2 (7\%) favored the iv. route. Pivot et al. in 2014 noted that reported AEs occurred in 292/479 (61\%) and 245/478 (51\%) of patients with early HER2-positive breast cancer during the pooled sc. and iv. periods, respectively $(\mathrm{p}<0.05)$ [60]. Similarly, Pivot et al. in 2017 reported AEs in $68 \%$ of patients with metastatic HER2-positive breast cancer receiving trastuzumab sc. and $44 \%$ of patients receiving trastuzumab iv. [61]. Among AEs observed with trastuzumab sc., $7 \%$ were grade $\geq 3$ and $3 \%$ were serious AEs. For trastuzumab iv., $4 \%$ were grade $\geq 3$ and $2 \%$ serious AEs. 

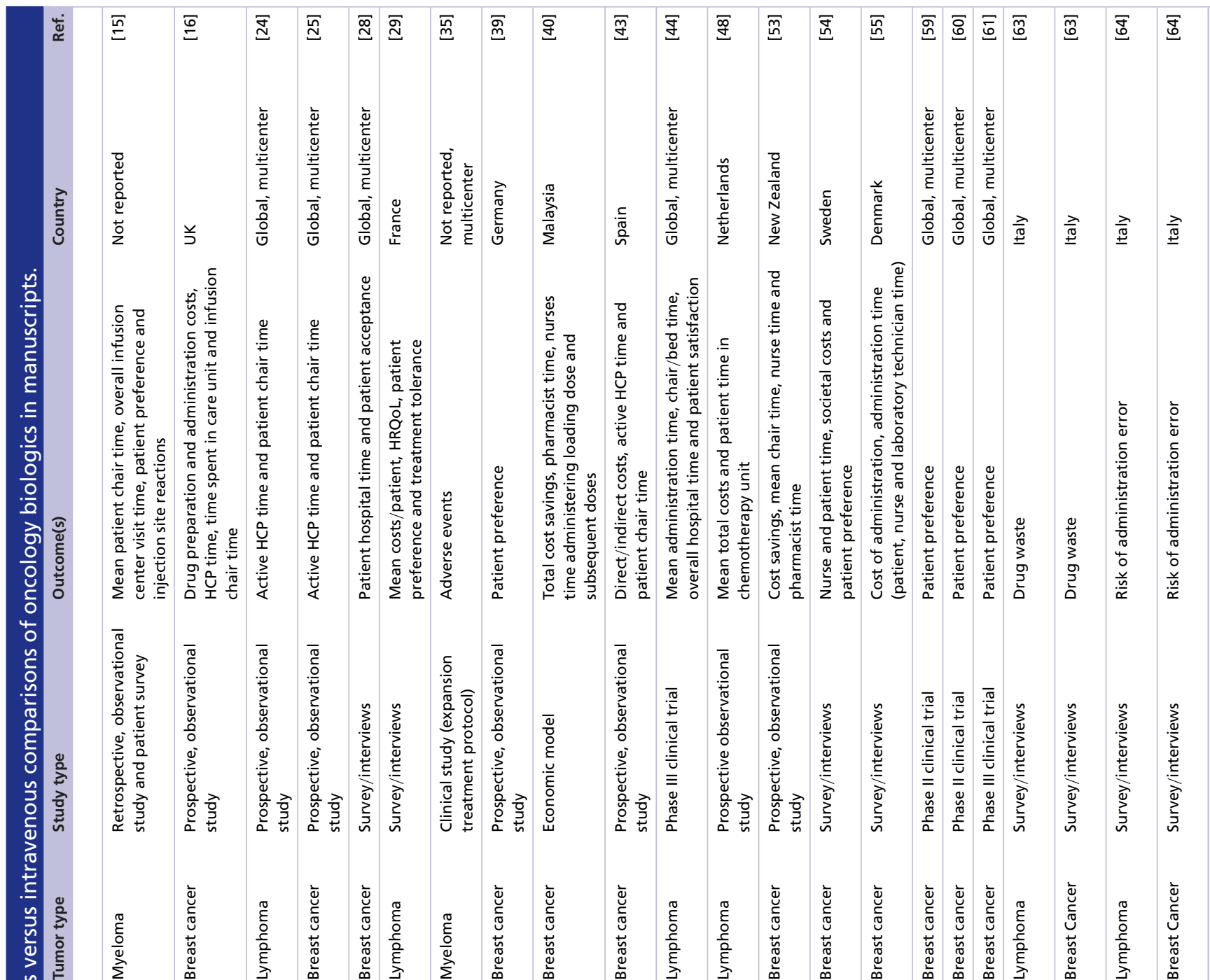

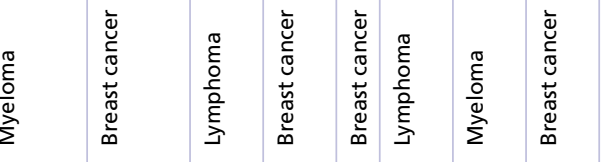
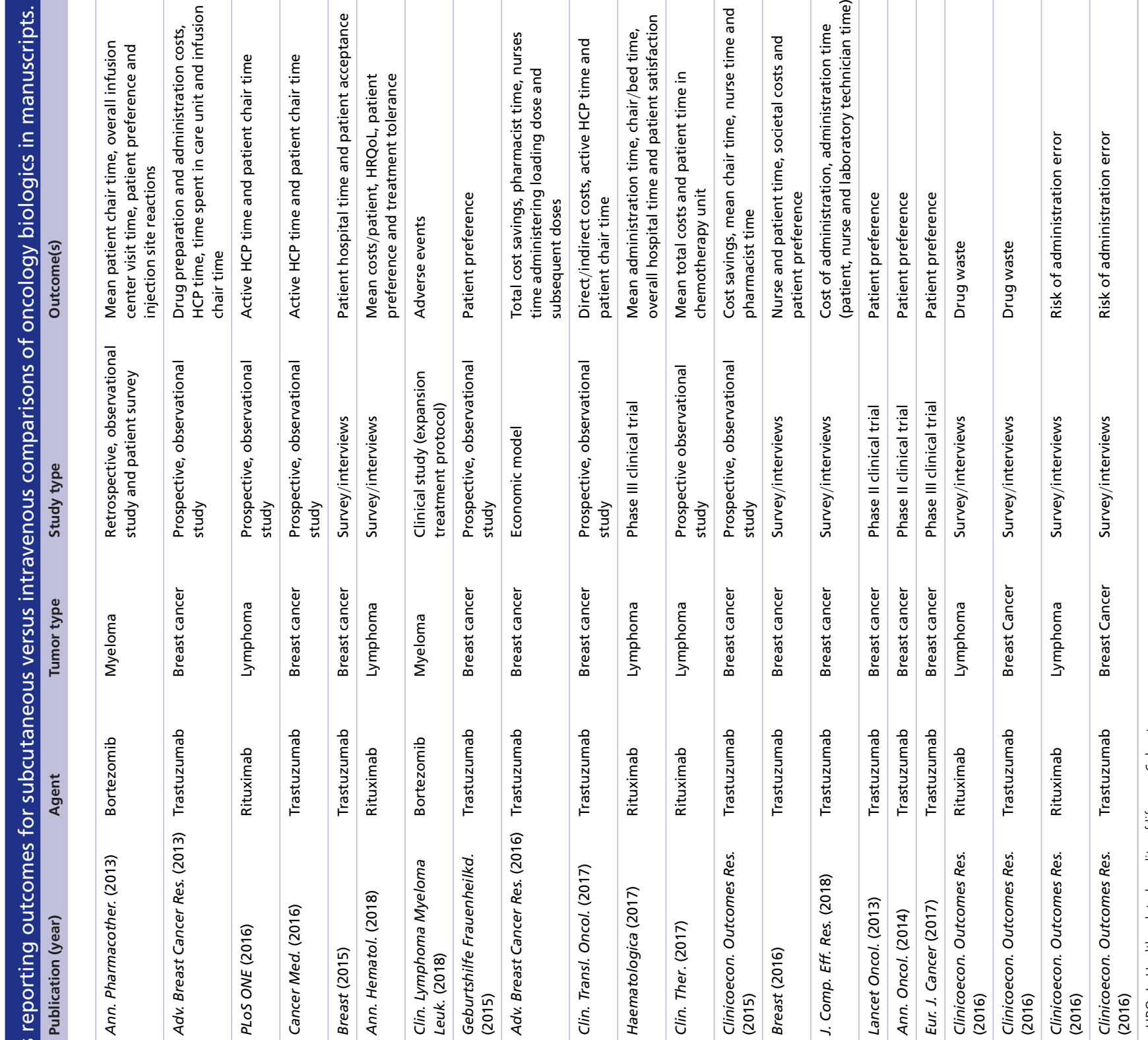


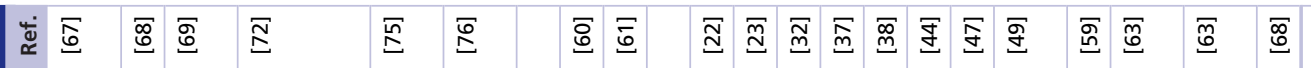

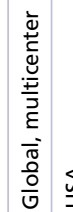

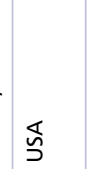

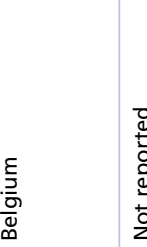

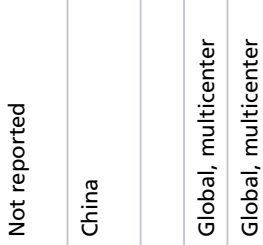

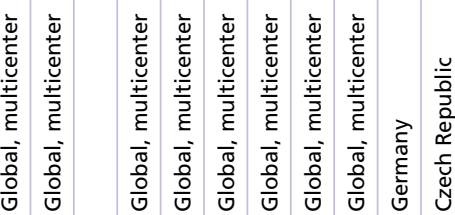
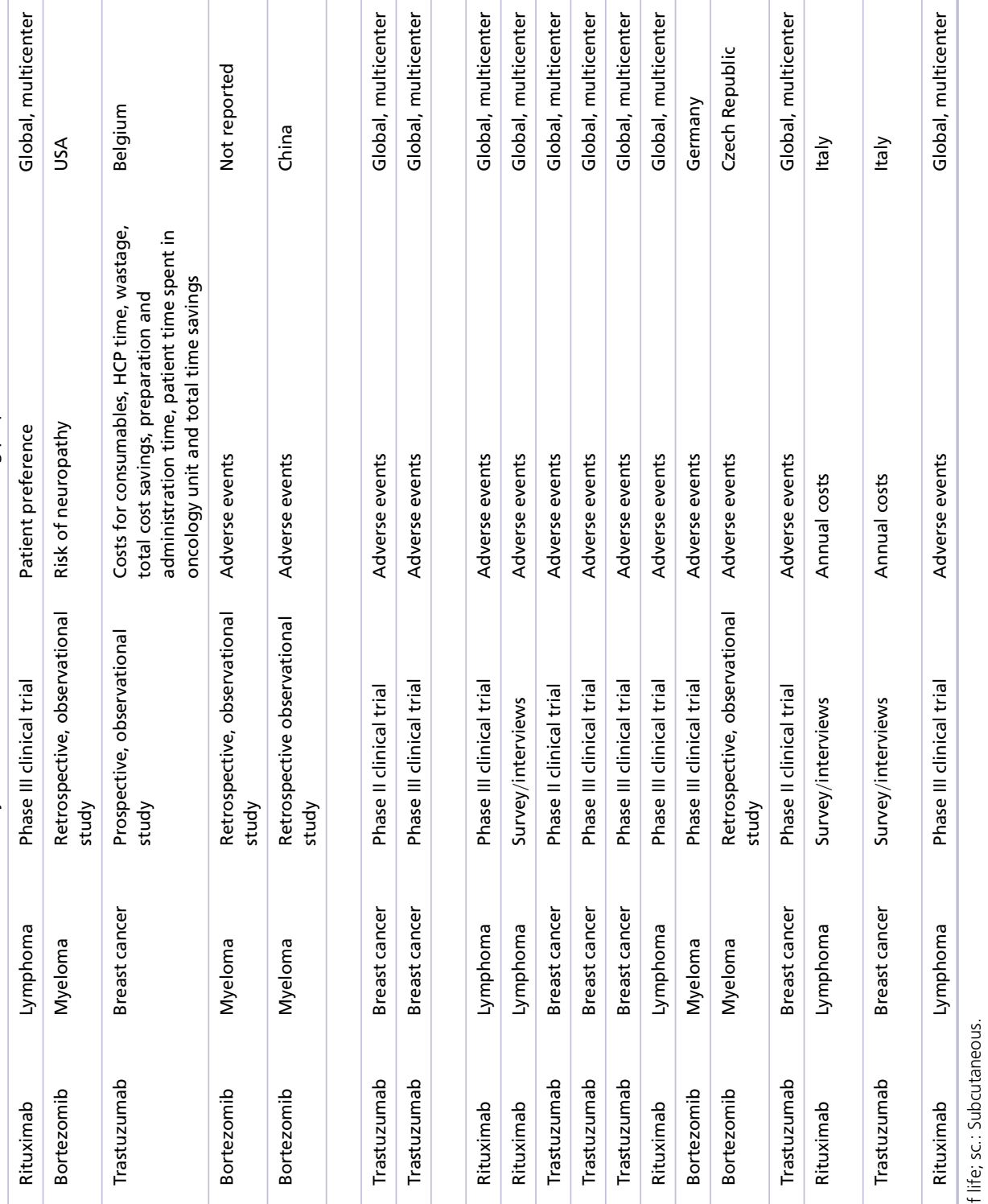
(A)

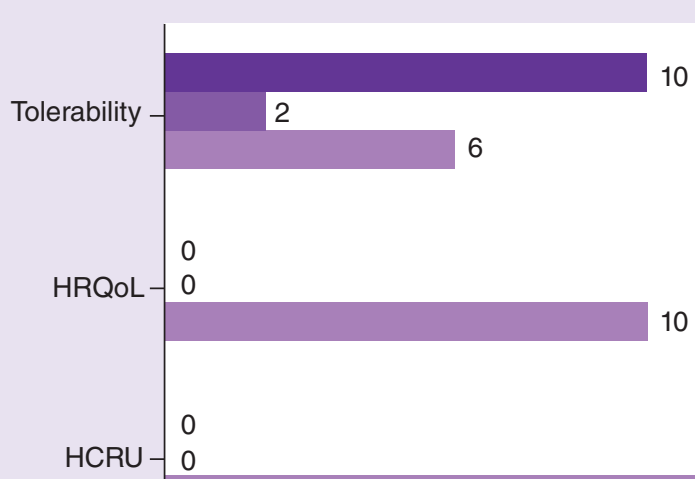

10

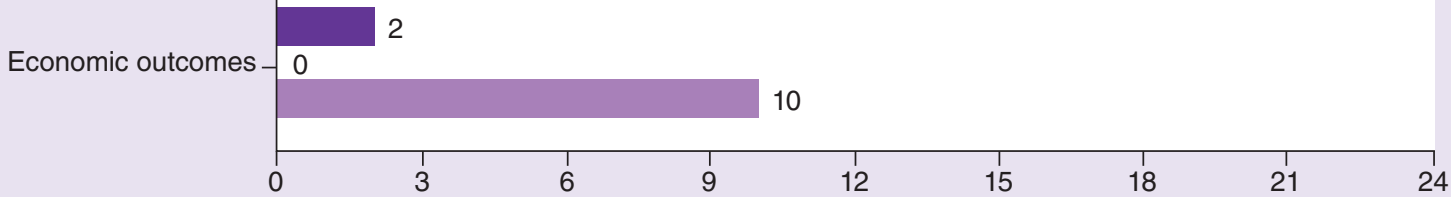

Not significant $\quad$ Favored iv. Favored sc.

(B)

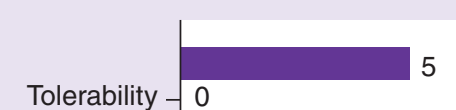

Tolerability $-0 \quad 7$

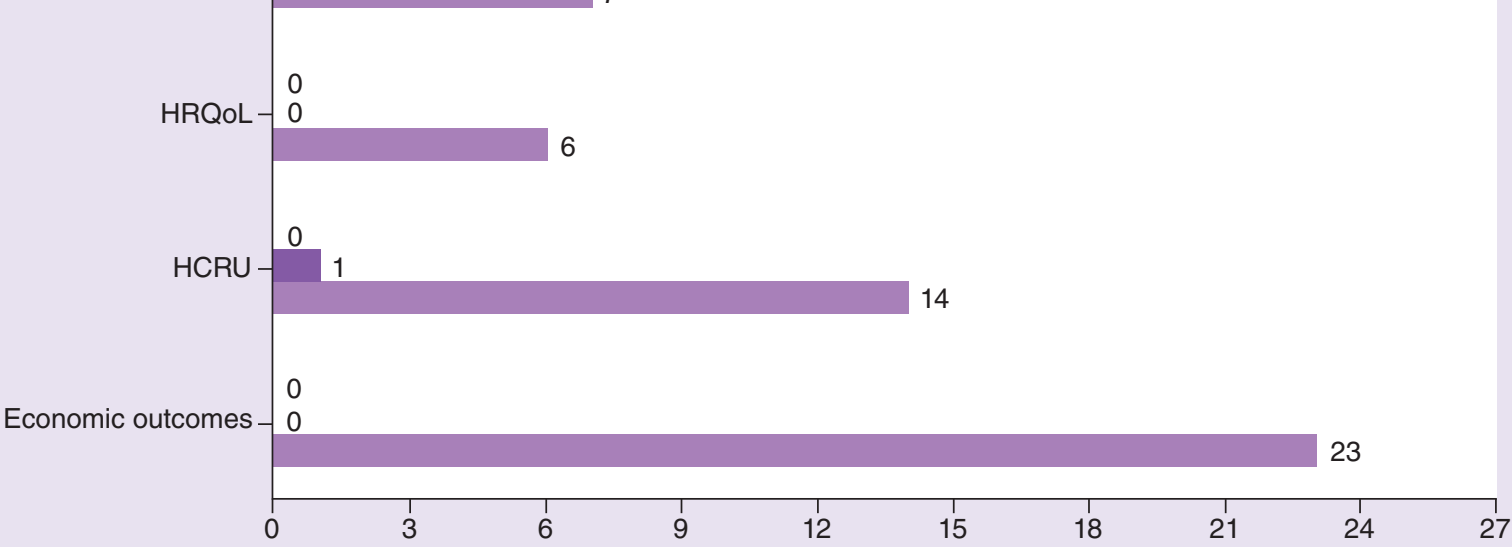

Not significant $\quad$ Favored iv. Favored sc.

Figure 2. Comparison of sc. versus iv. administration by outcome type. Outcomes reported in (A) manuscripts (61 outcomes in 33 manuscripts) and (B) congress abstracts (56 outcomes in 30 abstracts).

HCRU: Healthcare resource utilization; HRQoL: Health-related quality of life; iv.: Intravenous; sc.: Subcutaneous.

\section{HRQoL outcomes}

Sixteen $(25 \%)$ studies reported HRQoL outcomes, such as patient preference, assessed by surveys/interviews of patients treated in clinical practice or clinical trials. A range of questionnaires were used to collect HRQoL data, including the Rituximab Administration Satisfaction Questionnaire, the Patient Preference Questionnaire and the Cancer Therapy Satisfaction Questionnaire. One study used a general HRQoL instrument (EQ-5D-3L). Most of the studies (63\%) reporting HRQoL outcomes were manuscripts. All studies reported that patients preferred the sc. over the iv. administration route. 


\section{HCRU}

21 manuscripts and 15 abstracts reported HCRU outcomes. Most of these publications ( $90 \%$ of manuscripts, $87 \%$ of abstracts) reported HCP time as an outcome measure. All manuscripts and most abstracts (93\%) favored the sc. over the iv. route, while one abstract by Parinyanitikul et al. reported higher vial usage ( 14.7 vials for iv. and 18 vials for sc. administration of trastuzumab) from a cost-comparison analysis in a historical cohort [56]. Despite higher drug costs due to more wastage with trastuzumab sc. in this study, use of the sc. formulation over 18 treatment cycles resulted in an increase in free-bed time of 2.13 eight-hour days/patient compared with trastuzumab iv.. This saving was expected to provide a total of 1439 free-bed days for this historical patient cohort [56].

HCRU savings in terms of waste were addressed in one manuscript and one abstract, both reporting lower waste with sc. than iv. formulations [50,63]. Ponzetti et al. reported significant waste reductions (93-100\%) through sc. administration, with cost savings over the full treatment course of $€ 6057(\sim 6880)$ with rituximab sc. and $€ 28,399$ ( $\$ 32,258)$ with trastuzumab sc. versus iv. administration [63]. NA et al. also concluded that fixed-dose sc. formulation led to lower drug costs than the iv. formulation due to elimination of trastuzumab vial wastage [50].

Eight prospective, observational studies (seven manuscripts, one abstract) used a time-and-motion methodology to track efficiency of trastuzumab or rituximab administration among HCPs. All studies reported savings with sc. versus iv. administration. Mean active HCP time was substantially less for the sc. than the iv. route in these analyses (13-49 min for sc. vs 27-223 min for iv.), resulting in 31-79\% reductions in HCP time spent for sc. versus iv. administration. Mean patient chair time investigated in seven prospective, observational studies (six manuscripts, one abstract) decreased by $74-85 \%$ with sc. (11-70 $\mathrm{min})$ versus iv. (75-264 $\mathrm{min}$ ) administration.

\section{Economic outcomes}

Most of the manuscripts $(10[83 \%])$ and all abstracts (23 [100\%]) reporting economic outcomes favored the sc. administration route. The majority of studies reported direct medical costs such as the cost of drugs and consumables; only two abstracts focused on indirect costs alone [19,74]. Tomarchio et al. reported that patients who received rituximab sc. did not require day-hospital admission, allowing caregivers to spare 112 work days compared with caregivers of patients who received rituximab iv. [74] Chansung et al. reported a productivity loss for sc. and iv. rituximab of B8.66 ( $\sim 0.28)$ and B291.28 ( $\sim 9.29)$ bahts/cycle, respectively, in Thailand (daily wages $\sim \$ 10-\$ 38)[19]$.

In addition to evaluating direct costs, two studies reported drug wastage costs for the number of vials of sc. versus iv. product used [17,72]. Tjalma et al. found that iv. administration cost $€ 162.53(\sim \$ 184.62)$ more than the sc. route due to drug wastage (based on 65 administration episodes) [72]. Calvache et al. reported a 7\% average vial waste in the first treatment year and 3.5\% from the second year on [17]. Based upon this level of waste and direct drug cost differences, total modeled savings of $\$ 1,582,665$ were generated in 3 years and $\$ 2,296,620$ in 5 years for Ecuador, using the sc. route [17].

\section{Discussion}

In this REA, we evaluated evidence from reports published in the past 5 years comparing the humanistic, tolerability, economic and administrative impact of sc. versus iv. administration of biologics approved for the treatment of oncologic patients. Most of the outcomes reported in these publications favored the use of sc. over iv. administration for the agents identified in this analysis.

The retrieved publications focused predominantly on HCRU and economic outcomes, providing detailed evidence on overall direct/indirect costs and demonstrating resource and cost savings with the sc. compared with the iv. administration route. However, overall cost savings with sc. versus iv. administration may be potentially lower than reported for these anticancer biologics with the introduction of iv. biosimilars leading to a decrease in drug-related costs $[77,78]$.

HRQoL and HCRU advantages of sc. administration included patient preference and a reduced use of resources (i.e., vial wastage). Analysis of time-and-motion studies showed reductions in time spent in infusion chair by patients and in drug preparation/administration by HCPs, thus confirming the convenience of the sc. over iv. route. Further indirect cost savings may be associated with home administration of sc. biologics, potentially leading to increased patient compliance [21,79]. However, our focus was on HCP-administered treatments; home or patient-administered drugs were not part of the study objectives.

The review included studies from a fairly large number of countries $(>20)$, often conducted within a global, multicountry setting. Thus, this larger pool of study centers provided more generalizable information than studies 
from single countries/centers. Despite variations in healthcare systems and payer types across countries and centers, the findings were highly consistent for sc. administration being preferable over the iv. route for the evaluated outcomes. Studies from individual countries were relatively few (i.e., one study from the USA) [69]. Further research in this area might be useful to provide tailored information for multiple stakeholders (i.e., improved treatment experience for patients, greater administration efficiency for HCPs and cost savings for payers).

Some variability in methodology was evident across the publications analyzed, partly owing to differences in study types, which included Phase II or III clinical trials, prospective or retrospective studies, time-and-motion studies, economic models, surveys and interviews or questionnaires administered during clinical trials. Even across these diverse study types, there was consistency in the advantages identified with sc. versus iv. administration. Nonetheless, our findings may be limited by the use of a REA rather than a full systematic literature review with quality determination for each of the studies included in the analysis.

In terms of safety, prior clinical experience with bortezomib demonstrated a lower incidence of grade $\geq 2$ peripheral neuropathy with sc. versus iv. administration $(24 \mathrm{vs} 41 \%$; $\mathrm{p}=0.012)$ for relapsed multiple myeloma, indicating that sc. bortezomib may be preferable for patients at high risk or with pre-existing neuropathy $[6,80]$ In our analysis, the percentage of studies reporting reduced AEs with sc. versus iv. administration was slightly larger than the percentage reporting no statistically significant difference in AEs, and only two studies reported better tolerability with iv. administration. Both these studies, indicating a lower incidence of AEs with iv. versus sc. administration, reported findings with trastuzumab therapy in patients with early or metastatic HER2-positive breast cancer [60,61]. Patients receiving trastuzumab sc. experienced injection site reactions, pain and erythema (usually grade 1-2), which were the most frequent reason indicated by some patients (13\%) for preferring iv. over sc. administration of trastuzumab $[5,60]$. However, the overall safety profile of trastuzumab sc. was consistent with prior findings with trastuzumab iv. [60,61], as also recently confirmed in the final analysis of the HannaH Phase III trial in patients with early breast cancer, after a 6-year follow-up [81].

A more comprehensive analysis of all literature types, specifically clinical trials reporting detailed safety/tolerability outcomes, would be needed to confirm the potential tolerability benefits associated with sc. treatment of cancer patients, as suggested by this assessment of the published literature. The objective of this REA was to provide a qualitative description of the published data on selected outcomes and capture differences between sc. and iv. administration in everyday clinical practice rather than only within the confines of randomized clinical trials, which may have resulted in bias. Clinical trials were excluded unless they reported a HRQoL, HCRU or economic outcome. This restriction resulted in incomplete capture of tolerability outcomes, but was not likely to affect the economic, HCRU or HRQoL outcomes.

Furthermore, the majority of these studies reported results for rituximab and trastuzumab sc. which, differently from the iv. formulations, contain human recombinant hyaluronidase [82,83]. This could potentially impact the safety/tolerability profile, injection volume and time required for administration of these two sc. agents. Conversely, sc. administration of bortezomib does not require any additional agent compared with its equivalent iv. formulation and may, thus, be more representative of other biopharmaceuticals currently being administered sc. without the addition of hyaluronidase $[6-8]$.

Exploring the various studies according to patient subsets was challenging given the disparity in study sample classifications and baselines observed across the retrieved articles. Hence, parsing HCRU, economic, HRQoL or tolerability outcomes by patient characteristics was beyond the scope of this review and warrants further investigation.

In conclusion, real-world evidence shows that sc. administration of biologics to oncologic patients saves provider and facility time through savings in drug preparation and administration time; these, in turn, result in lower costs for sc. than for iv. administration of the same agent. Patients also reported a better quality of life and a preference for sc. than iv. therapy. All together, these findings support further development of sc. formulations for anti-PD1/PD-L1 antibodies [7-9], which are increasingly showing improved outcomes in patients with various malignancies as single-agent or combination therapies [82-87. In view of the expected increase in the global use of these therapies across cancer indications and in high numbers of patients, the future availability of anti-PD-1/PD-L1 antibodies for sc. administration may be beneficial for patients, HCPs and payers, with potential cost/resource savings and improved treatment delivery. 


\section{Future perspective}

All together, findings from this assessment of published evidence on the humanistic and economic impact of sc. versus iv. administration of approved cancer therapies support active development of novel sc. formulations for oncology biologics, in the effort to provide useful therapeutic options. sc. formulations of PD-1/PD-L1 inhibitors, in particular, are expected to be of substantial importance in the upcoming years, in view of the increasing global use of these therapies across multiple cancer indications and large patient populations.

\section{Executive summary}

- Anticancer agents approved for both subcutaneous (sc.) and intravenous (iv.) administration in patients with cancer currently include the anti-CD20 antibody rituximab, the anti-HER2 antibody trastuzumab and the proteasome inhibitor bortezomib.

- Novel sc. formulations being investigated in clinical studies include anti-PD-1/PD-ligand 1 antibodies, pertuzumab, bevacizumab and daratumumab, across multiple indications.

- The objective of this rapid evidence assessment (REA) was to evaluate and discuss evidence published in the past 5 years on the humanistic and economic impact of sc. versus iv. administration of cancer therapies approved for the treatment of patients with solid or hematologic malignancies.

- Based on the inclusion criteria, the publications assessed in this REA reported mostly findings on healthcare resource utilization and economic outcomes, providing detailed evidence on direct and indirect costs.

- Overall, of 61 outcomes compared in 33 manuscripts, 47 (77\%) favored sc., 2 (3\%) favored iv. and 12 (20\%) showed no statistically significant difference between sc. and iv. administration.

- Of 56 outcomes compared in 30 congress abstracts, 50 (89\%) favored sc., 1 (2\%) favored iv. and 5 ( $9 \%$ ) showed no statistically significant difference between sc. and iv. administration.

- Study results demonstrated resource and cost savings with sc. compared with iv. administration. Analysis of time-and-motion studies showed reductions in time spent in infusion chair and in caregiver assistance, as well as in time spent for drug preparation and administration by healthcare providers.

- In terms of tolerability, the percentage of studies reporting reduced adverse events with sc. versus iv. administration was slightly higher than those reporting no statistically significant difference, and only two trastuzumab-related studies favored iv. administration.

- Evaluation of patients reported outcomes demonstrated a better health-related quality of life with sc. versus iv. biologic therapy and a preference among most patients for sc. administration.

- The humanistic and economic advantages identified in this REA for sc. versus iv. administration of oncology biologics for patients, healthcare providers and payers appeared to be consistent across the diverse study methodologies and study types reported.

\section{Supplementary data}

To view the supplementary data that accompany this paper please visit the journal website at: https://www.futuremedicine.com/d oi/suppl/10.2217/fon-2019-0368

\section{Author contributions}

All authors contributed to the design and development of this work, data analysis and interpretation and manuscript writing. All authors approved final version of the manuscript.

Financial \& competing interests disclosure

KC Anderson is consultant for Bristol-Myers Squibb, Celgene, Gilead, Janssen and Millennium-Takeda. O Landgren has disclosed grant support from the National Institutes of Health (NIH), Food and Drug Administration (FDA), Leukemia and Lymphoma Society (LLS), Multiple Myeloma Research Foundation (MMRF), IMF, Perelman Family Foundation, Rising Tides Foundation, Amgen, Celgene, Glenmark, Janssen, Karyopharm, Seattle Genetics and Takeda; advisory boards/honoraria from Adaptive, Amgen, Binding Site, Bristol-Myers Squibb, Celgene, Cellectis, Glenmark, Janssen, Juno and Pfizer; and participation in Independent Data Monitoring Committees for Janssen, Merck and Takeda. RC Arend has disclosed advisory board honoraria from AstraZeneca, Clovis, Pfizer and Tesaro. J Chou and IA Jacobs are employees of Pfizer and hold stock/stock options in Pfizer. The authors have no other relevant affiliations or financial involvement with any organization or entity with a financial interest in or financial conflict with the subject matter or materials discussed in the manuscript apart from those disclosed.

Medical writing was provided by S Mariani, of Engage Scientific Solutions and was funded by Pfizer. 
Open access

This work is licensed under the Attribution-NonCommercial-NoDerivatives 4.0 Unported License. To view a copy of this license, visit http://creativecommons.org/licenses/by-nc-nd/4.0/

\section{References}

Papers of special note have been highlighted as: $\bullet$ of interest; $\bullet \bullet$ of considerable interest

1. Awwad S, Angkawinitwong U. Overview of antibody drug delivery. Pharmaceutics 10, pii:E83 (2018).

2. Bittner B, Richter W, Schmidt J. Subcutaneous administration of biotherapeutics: an overview of current challenges and opportunities. BioDrugs 32(5), 425-440 (2018).

3. Viola M, Sequeira J, Seiça R et al. Subcutaneous delivery of monoclonal antibodies: how do we get there? J. Control. Rel. 286, 301-314 (2018).

4. Prescribing Information. Rituxan Hycela (rituxan and hyaluronidase human) injection, for subcutaneous use (2017). https://www.gene.com/download/pdf/rituxan_hycela_prescribing.pdf

5. Prescribing Information. Herceptin Hylecta (trastuzumab and hyaluronidase-oysk) injection, for subcutaneous use (2019). https://www.gene.com/download/pdf/herceptin_hylecta_prescribing.pdf

6. Prescribing Information. Velcade (bortezomib) for injection, for cutaneous or intravenous use (2017). http://www.velcade.com/Files/PDFs/Velcade_Prescribing_Information.pdf

7. Johnson ML, Braiteh F, Grilley-Olson JE et al. Assessment of subcutaneous vs intravenous administration of anti-PD-1 antibody PF-06801591 in patients with advanced solid tumors: a Phase I dose-escalation trial. JAMA Oncol. 5(7), 999-1007 (2019).

8. Xu J, Qin S, Zhang Y et al. A Phase I, open, multiple dose, dose escalation and expansion study to evaluate the safety, tolerability, and pharmacokinetics of KN035 (anti-PD-L1 antibody) administered in subcutaneous injection as a single agent to Chinese subjects with advanced solid tumors. J. Clin. Oncol. 36(Suppl. 15), e15138 (2018).

9. Papadopoulos KP, Harb W, Lu N et al. Phase I study of KN035, a novel fusion anti-PD-L1 antibody administered subcutaneously in patients with advanced solid tumors in the USA. Ann. Oncol. 29(Suppl. 8), 1140PD (2018).

10. Kirschbrown WP, Wynne C, Kågedal M et al. Development of a subcutaneous fixed-dose combination of pertuzumab and trastuzumab: results from the Phase Ib dose-finding study. J. Clin. Pharmacol. 59(5), $702-716$ (2019).

11. Quillin J, Read WL. A Phase II study of subcutaneous bevacizumab for recurrent glioblastoma: roadblocks and promises. J. Clin. Oncol. 36(Suppl. 15), e14089 (2018).

12. Chari A, Nahi H, Mateos M-V et al. Subcutaneous delivery of daratumumab in patients (pts) with relapsed or refractory multiple myeloma (RRMM): PAVO, an open-label, multicenter, dose escalation Phase Ib study. Blood 130(Suppl. 1), 838 (2017).

13. Shamseer L, Moher D, Clarke M et al. PRISMA-P group: preferred reporting items for systematic review and meta-analysis protocols (PRISMA-P) 2015: elaboration and explanation. BMJ350, g7647 (2015).

14. Bacchiarri F, Nozzoli C, Antonioli E et al. Safety and efficacy of SC versus IV bortezomib in patients with newly elderly diagnosed multiple myeloma. Haematologica 102(Suppl. 3), 167 (2017).

15. Barbee MS, Harvey RD, Lonial S et al. SC versus IV bortezomib: efficiency practice variables and patient preferences. Ann. Pharmacother. 47(9), 1136-1142 (2013).

16. Burcombe R, Chan S, Simcock R et al. SC Trastuzumab (Herceptin ${ }^{\circledR}$ ): a UK time and motion study in comparison with IV formulation for the treatment of patients with HER2-positive early breast cancer. Adv. Breast Cancer Res. 2, 133-140 (2013).

17. Calvache J, Briceno V. Budget impact analysis of the use of trastuzumab SC in the treatment of HER2 positive in public health institutions of Ecuador. Value Health 20, A130 (2017).

18. Castillo-Fernandez O, Cabreja A, Arauz E et al. Do patients and nurses outside clinical trial prefer SC trastuzumab over conventional IV infusion? Instituto Oncologico Nacional experience. Cancer Res. 77(Suppl. 4), P5-11-16 (2017).

19. Chansung K, Chuncharunee S, Khuhapinant A et al. Preference of rituximab SC (R-SC) in Thai patients with diffused large B-cell lymphoma (DLBCL) and follicular lymphoma (FL): a Thai context. Value in Health 21(Suppl. 2), S17 (2018).

20. Choi MH, Lim S, Wong A. Cost-minimisation analysis of SC vs IV rituximab for the treatment of follicular lymphoma and diffuse large B-cell lymphoma in Singapore. Value in Health 21(Suppl. 2), S16 (2018)

21. Cocquyt VF, Martinez-Mena CL, Martens MT et al. BELIS: safety and tolerability of at home administration of trastuzumab (Herceptin) SC for the treatment of patients with HER2-positive early breast cancer. Cancer Res. 77(4; Suppl. 1), P4-21-17 (2017).

22. Davies A, Merli F, Mihaljević B et al. Efficacy and safety of SC rituximab versus IV rituximab for first-line treatment of follicular lymphoma (SABRINA): a randomised, open-label, Phase III trial. Lancet Haematol. 4(6), e272-e282 (2017).

23. Davies A, Merli F, Mihaljevic B et al. Pharmacokinetics and safety of SC rituximab in follicular lymphoma (SABRINA): stage 1 analysis of a randomised Phase III study. Lancet Oncol. 15(3), 343-352 (2014).

24. De Cock E, Kritikou P, Sandoval M et al. Time savings with rituximab SC injection versus rituximab IV infusion: a time and motion study in eight countries. PLoS ONE 11(6), e0157957 (2016). 
25. De Cock E, Pivot X, Hauser N et al. A time and motion study of SC versus IV trastuzumab in patients with HER2-positive early breast cancer. Cancer Med. 5(3), 389-397 (2016).

26. Di Rocco A, Scerbo G, Ansuinelli M et al. Efficacy, safety and cost analysis of SC vs IV rituximab in patients with diffuse large B-cell lymphoma treated with RCHOP. Haematologica 3, 110-111 (2017).

27. Dumitru D, Benson J, Wishart G et al. Rates of ipsilateral breast tumor recurrence (IBTR) following breast conserving surgery (BCS) and hypofractionated radiotherapy for ductal carcinoma in situ (DCIS). Cancer Res. 77(4 Suppl. 1), P1-11-03 (2017).

28. Fallowfield L, Osborne S, Langridge C et al. Implications of SC or IV delivery of trastuzumab; further insight from patient interviews in the PrefHer study. Breast 24(2), 166-170 (2015).

29. Fargier E, Ranchon F, Huot L et al. SMABcare study: SC monoclonal antibody in cancer care: cost-consequence analysis of SC rituximab in patients with follicular lymphoma. Ann. Hematol. 97(1), 123-131 (2018).

30. Franken MG, Kanters TA, Coenen JL et al. Saving healthcare and societal costs by changing the route of administration of oncology drugs. Value in Health 21(Suppl. 3), S28 (2018)

31. Ghosh W, Lim S, Wong A. Cost-minimisation analysis of SC versus IV trastuzumab for the treatment of early breast cancer and metastatic breast cancer in Singapore. Value in Health 21(Suppl. 2), S16 (2018)

32. Gligorov J, Curigliano G, Müller V et al. Switching between IV and SC trastuzumab: safety results from the PrefHer trial. Breast 34 , 89-95 (2017).

33. Gomes G, Ho R, Rufino C et al. Budget impact analysis of rituximab IV versus SC from public Brazilian hospital. ISPOR 22nd Annual International Meeting. Boston, MA (2017).

34. Hansen RN, Wallick CJ, Sullivan SD. Budget impact of the introduction of SC rituximab to U.S. health plans. Value Health 21(Suppl. 1), S22-S23 (2018).

35. Hansen VL, Coleman M, Elkins S et al. An expanded treatment protocol of panobinostat plus bortezomib and dexamethasone in patients with previously treated myeloma. Clin. Lymphoma Myeloma Leuk. 18(6), 400-407 (2018).

36. Irwin $\mathrm{S}$, Rowntree $\mathrm{C}$, Cosh $\mathrm{H}$ et al. Positive benefits of changing from IV rituximab administration to SC administration: a single centre experience. Br. J. Hematol. 176(Suppl. 1), 68-69 (2017).

37. Jackisch C, Hegg R, Stroyakovskiy D et al. HannaH Phase III randomised study: association of total pathological complete response with event-free survival in HER2-positive early breast cancer treated with neoadjuvant-adjuvant trastuzumab after 2 years of treatment-free follow-up. Eur. J. Cancer 62, 62-75 (2016).

38. Jackisch C, Kim SB, Semiglazov V et al. SC versus IV formulation of trastuzumab for HER2-positive early breast cancer: updated results from the Phase III HannaH study. Ann. Oncol. 26(2), 320-325 (2015).

39. Jackisch C, Müller V, Dall P et al. SC trastuzumab for HER2-positive breast cancer - evidence and practical experience in 7 German centers. Geburtshilfe Frauenheilkd. 75(6), 566-573 (2015).

40. Lee WC, Haron MR, Yu KL et al. Economic analysis of IV vs. SC administered trastuzumab for the treatment of HER2+ early breast cancer in Malaysia. Adv. Breast Cancer Res. 5(1), 1-13 (2016).

41. Lewis P, Jones H, Skelley K et al. Switching to SC trastuzumab administration: quantifying the benefits. Clin. Oncol. 29, E101 (2017).

42. Lopez MA, Samanes MA, Tena IP et al. Switching from IV to SC formulation of trastuzumab: costs and safety. Eur. J. Hosp. Pharm. 24(Suppl. 1), A57 (2017).

43. Lopez-Vivanco G, Salvador J, Diez R et al. Cost minimization analysis of treatment with IV or SC trastuzumab in patients with HER2-positive breast cancer in Spain. Clin. Transl. Oncol. 19(2), 1454-1461 (2017).

- Evaluation of cost efficiencies with subcutaneous (sc.) versus intravenous therapy in patients with breast cancer.

44. Lugtenburg P, Avivi I, Berenschot $\mathrm{H}$ et al. Efficacy and safety of SC and IV rituximab plus cyclophosphamide, doxorubicin, vincristine, and prednisone in first-line diffuse large B-cell lymphoma: the randomized MabEase study. Haematologica 102(11), 1913-1922 (2017).

45. Martin C, Alcedo J, Araúz E. Comparative analysis of costs between SC formulation of trastuzumab versus IV formulation from the perspective of the Instituto Oncológico Nacional of Panamá from January to December 2016. Cancer Res. 78(Suppl.4), P4-12-13 (2018).

46. Marty BC, Blein C, Borg MC et al. A multi-center evaluation of clinical pathways cost and time using real-life data in 411 breast cancer patients treated with IV versus SC trastuzumab. Eur. J. Cancer 92(Suppl. 3), S106-S107 (2018).

47. Merz M, Salwender H, Haenel M et al. SC versus IV bortezomib in two different induction therapies for newly diagnosed multiple myeloma: an interim analysis from the prospective GMMG-MM5 trial. Haematologica 100(7), 964-969 (2015).

48. Mihajlović J, Bax P, van Breugel E et al. Microcosting study of rituximab SC injection versus IV infusion. Clin. Ther. 39(6), 1221-1232 (2017).

49. Minarik J, Pavlicek P, Pour L et al. Czech Myeloma Group: SC bortezomib in multiple myeloma patients induces similar therapeutic response rates as IV application but it does not reduce the incidence of peripheral neuropathy. PLoS ONE 10(4), e0123866 (2015).

50. NA Ali A, Al-Tweigeri T, Zekri J et al. Financial impact of introducing SC trastuzumab (herceptin) versus currently used IV trastuzumab (herceptin) on the budgets of different hospitals across Kingdom of Saudi Arabia (KSA). Value Health 20(9), A422 (2017).

51. Nierenberger A, Gessier F, Forges F et al. SC trastuzumab versus IV trastuzumab: an impact study. Eur. J. Hosp. Pharm. 24, A216 (2017). 
52. Nikolov O, Sterjev Z, Dimovski A et al. Cost-minimization analysis of rituximab SC formulation versus IV administration of rituximab for the treatment of non-Hodgkin's lymphoma in the Republic of Macedonia. Haematologica 102(Suppl. 2), 600 (2017).

53. North RT, Harvey VJ, Cox LC et al. Medical resource utilization for administration of trastuzumab in a New Zealand oncology outpatient setting: a time and motion study. Clinicoecon. Outcomes Res. 7, 423-430 (2015).

54. Olofsson S, Norrlid H, Karlsson E et al. Societal cost of SC and IV trastuzumab for HER2-positive breast cancer - an observational study prospectively recording resource utilization in a Swedish healthcare setting. Breast 29, 140-146 (2016).

55. Olsen J, Jensen KF, Olesen DS et al. Costs of SC and IV administration of trastuzumab for patients with HER2-positive breast cancer. J. Comp. Eff. Res. 7(5), 411-419 (2018).

56. Parinyanitikul N, Lochid-Amnuay S. A historical cohort for cost comparison analysis of IV versus SC trastuzumab in Thailand. Value in Health 21(Suppl. 2), S12 (2018).

57. Paszkiewicz-Kozik E, Dlugosz-Danecka M, Robak PJ et al. SC compared to IV administration of rituximab plus CHOP in patients with diffuse large B-cell lymphoma - a study by the Polish Lymphoma Research Group. Blood 130, 5217 (2017).

58. Petrakova K, Melichar B, Bortlicek Z et al. Preference of trastuzumab administration route (IV or SC) in patients in the Czech Republic. Cross-sectional study on 429 patients. Cancer Res. 77(Suppl.4), P4-21-12 (2017).

59. Pivot X, Gligorov J, Müller V et al.; PrefHer Study Group. Preference for SC or IV administration of trastuzumab in patients with HER2-positive early breast cancer (PrefHer): an open-label randomised study. Lancet Oncol. 14(10), 962-970 (2013).

60. Pivot X, Gligorov J, Müller V et al.; PrefHer Study Group. Patients' preferences for SC trastuzumab versus conventional IV infusion for the adjuvant treatment of HER2-positive early breast cancer: final analysis of 488 patients in the international, randomized, two-cohort PrefHer study. Ann. Oncol. 25(10), 1979-1987 (2014).

- Study reporting patient outcomes with sc. administration of trastuzumab in early breast cancer.

61. Pivot X, Spano JP, Espie M et al. Patients' preference of trastuzumab administration (SC versus IV) in HER2-positive metastatic breast cancer: results of the randomised MetaspHer study. Eur. J. Cancer 82, 230-236 (2017).

- Study reporting patient outcomes with sc. administration of trastuzumab in metastatic breast cancer.

62. Pombo FH, Passos PM, Gamboa NF et al. Safety profile and reduced observation time in outpatients using SC herceptin. J. Clin. Oncol. 36(Suppl. 15), e18848 (2018).

63. Ponzetti $\mathrm{C}$, Canciani $\mathrm{M}$, Farina $\mathrm{M}$ et al. Potential resource and cost saving analysis of SC versus IV administration for rituximab in non-Hodgkin's lymphoma and for trastuzumab in breast cancer in 17 Italian hospitals based on a systematic survey. Clinicoecon. Outcomes Res. 8, 227-233 (2016).

64. Ponzetti C, Canciani M, Rarina $\mathrm{M}$ et al. Administrative risk quantification of SC and IV therapies in Italian centers utilizing the failure mode and effects analysis approach. Clinicoecon. Outcomes Res. 8, 353-359 (2016).

65. Poquet-Jornet JE, Carrera Hueso J, Crespo C et al. Budget impact analysis with use of subcutaneus trastuzumab. ISPOR Europe, Barcelona, Spain (2018) PCN80.

66. Rauf M, Dada R, Awad N, Safwat M et al. To determine the financial impact of introducing SC rituximab (MABTHERA) vs. currently used IV rituximab (MABTHERA) on the budgets of different hospitals across the kingdom of Saudi Arabia (KSA). Value Health 20(9), A424 (2017).

67. Rule S, Collins GP, Samanta K. SC vs IV rituximab in patients with non-Hodgkin lymphoma: a time and motion study in the United Kingdom. J. Med. Econ. 17(7), 459-468 (2014).

- Analysis of effects and advantages with rituximab sc. treatment in patients with lymphoma.

68. Rummel M, Kim TM, Aversa F et al. Preference for SC or IV administration of rituximab among patients with untreated CD20 ${ }^{+}$diffuse large B-cell lymphoma or follicular lymphoma: results from a prospective, randomized, open-label, crossover study (PrefMab). Ann. Oncol. 28(4), 836-842 (2017).

69. Sidana S, Narkhede M, Elson P et al. Neuropathy and efficacy of once weekly SC bortezomib in multiple myeloma and light chain (AL) amyloidosis. PLoS ONE 12(3), e0172996 (2017).

- Study demonstrating improved safety with sc. versus intravenous administration of bortezomib in patients with hematologic malignancies.

70. Stojanovik A, Cvetanovska L, Chevreska L et al. Preference of Macedonian NHL patients for SC vs IV administration of rituximab. Leukemia Res. 61, S32 (2017).

71. Tjalma WAA, Van Den Mooter T, Mertens T et al. Trastuzumab IV versus SC: a time, motion and cost assessment in a lean operating day care oncology unit. Cancer Res. 77(4 Suppl.), P-4-21-15 (2017).

72. Tjalma WAA, Van den Mooter T, Mertens T et al. SC trastuzumab (Herceptin) versus IV trastuzumab for the treatment of patients with HER2-positive breast cancer: a time, motion and cost assessment study in a lean operating day care oncology unit. Eur. J. Obstet. Gynecol. Reprod. Biol. 221, 46-51 (2018).

73. Todorovic V, Durutovic I, Ivanovska A et al. SC vs IV administration of trastuzumab in HER2+ breast cancer patients: a Montenegrin cost-minimization analysis. Value Health 20(9), A443 (2017). 
74. Tomarchio V, Surano MA, Tafuri MA et al. Management, economic and social impact of sub-cutaneous rituximab administration in lymphoproliferative malignancies. Haematologica 102, 298 (2017).

75. Wu S, Zheng C, Chen S et al. SC Administration of bortezomib in combination with thalidomide and dexamethasone for treatment of newly diagnosed multiple myeloma patients. Biomed. Res. Int. 2015, 927105 (2015).

76. Xu Y, Deng S, Mao X et al. Tolerance, kinetics, and depth of response for SC versus IV administration of bortezomib combination in chinese patients with newly diagnosed multiple myeloma. Clin. Lymphoma Myeloma Leuk. 18(6), 422-430 (2018).

77. Rifkin RM, Peck SR. Biosimilars: implications for clinical practice. J. Oncol. Pract. 13(Suppl. 9), 24S-31S (2017).

78. Renwick MJ, Smolina K, Gladstone EJ, Weymann D, Morgan SG. Postmarket policy considerations for biosimilar oncology drugs. Lancet Oncol. 17(1), e31-e38 (2016).

79. Wolfromm A, Mittaine B, Gandrille $\mathrm{N}$ et al. Home administration of subcutaneous rituximab is safe and associated with significant cost saving: a single center experience. Blood 130(Suppl. 1), 4676 (2017).

80. Moreau P, Pylypenko H, Grosicki $S$ et al. Subcutaneous versus intravenous administration of bortezomib in patients with relapsed multiple myeloma: a randomised, Phase III, non-inferiority study. Lancet Oncol. 12(5), 431-440 (2011).

81. Jackisch C, Stroyakovskiy D, Pivot X et al. Subcutaneous vs intravenous trastuzumab for patients with ERBB2-positive early breast cancer final analysis of the HannaH Phase III randomized clinical trial. JAMA Oncol. 5(5), e190339 (2019).

82. Davies A, Berge C, Boehnke A et al. Subcutaneous rituximab for the treatment of B-cell hematologic malignancies: a review of the scientific rationale and clinical development. Adv. Ther. 34(10), 2210-2231 (2017).

83. Pimentel F, Morgan G, Tiezzi DG et al. Development of new formulations of biologics: expectations, immunogenicity, and safety for subcutaneous trastuzumab. Pharmaceut. Med. 32(5), 319-325 (2018).

84. Balar AV, Weber JS. PD-1 and PD-L1 antibodies in cancer: current status and future directions. Cancer Immunol. Immunother. 66(5), 551-564 (2017).

85. Gong J, Chehrazi-Raffle A, Reddi S et al. Development of PD-1 and PD-L1 inhibitors as a form of cancer immunotherapy: a comprehensive review of registration trials and future considerations. J. Immunother. Cancer 6(1), 8 (2018).

-. Overview of safety and efficacy of treatment with approved PD-1/PD-L 1 inhibitors, across a wide range of tumor types and clinical settings.

86. Sanmamed MF, Chen L. A paradigm shift in cancer immunotherapy: from enhancement to normalization. Cell 175(2), 313-326 (2018).

87. Keung EZ, Wargo JA. The current landscape of immune checkpoint inhibition for solid malignancies. Surg. Oncol. Clin. N. Am. 28(3), 369-386 (2019). 
